\title{
Arthroscopic Treatment of Chronic Patellar Tendinopathy "Jumper's Knee"
}

\begin{abstract}
a
Department of orthopedic surgery, faulty of medicine, Kafrelsheikh University, Kafrelsheikh, Egypt.

${ }^{b}$ Department of orthopedic surgery, Benha faculty of medicine ,Benha university, Egypt.
\end{abstract}

Ahmed R. Elzeiny ${ }^{a}$, Adel H. Adawy ${ }^{\text {b }}$, Hatem E. Elgohary ${ }^{\text {a }}$, Saad A. Shoulah ${ }^{\text {b }}$

\section{Abstract:}

Correspondence to: Ahmed R. Elzeiny, Department of orthopedic surgery, faulty of medicine, Kafrelsheikh University, Kafrelsheikh, Egypt

Email:

elzeiny1890@gmail.com

Received: 2 August 2019

Accepted :6 November 2019
Background: Jumper's knee, patellar tendinopathy, is a clinical term used to describe patellar tendon pain, swelling, and/or dysfunction resulting from acute injury or, more commonly, chronic overuse. This condition is prevalent among athletes. In patients with patellar tendinopathy in whom non-operative management is unsuccessful; surgery is an option to return to high levels of physical activity. Aim: This work aimed to evaluate the results of arthroscopic management of cases with chronic refractory patellar tendinopathy (jumper's knee). Methods: This is a prospective study that was done at Kafr El-Sheikh and Benha university hospital. 28 patients involved in sports activities with chronic patellar tendinitis aged 18-3o years with the mean age of 23.25 years were considered to have arthroscopic treatment to refractory chronic patellar tendinopathy after at least 3 months of conservation. All patients in the study underwent arthroscopic debridement of the Hoffa's bad fat, debridement of abnormal patellar tendon, with or without excision of the lower pole of the patella. Preoperative and postoperative evaluation was undertaken using the VAS score, Lysholm Scores and Victorian Institute of Sport Assessment-Patella (VISA-P) scores for all patients. No patients were lost to follow-up. Results: The VAS, Lysholm, and VISA-P scores all significantly improved at last follow-up. There were no postoperative complications. 27 patients of 28 were able to perform at their preinjury sports activities. One case was not able to return to preinjury sports activity. Conclusion: arthroscopic surgery for patients with patellar tendinopathy, refractory to non-operative management, appears to provide significant improvements in symptoms and function.

Key-words: Jumper's knee, Arthroscopic treatment, Patellar tendon. 


\section{Introduction}

Jumper's knee, or patellar tendinopathy, is a clinical term used to describe patellar tendon pain, swelling, and/or dysfunction resulting from acute injury or, more commonly, chronic overuse. It was originally described by Blazina in 1973 and named as jumper's knee due to its association with athletes whose sport requires frequent jumping. ${ }^{(1)}$

A number of theories have been suggested for the pathogenesis of the anterior knee pain in patellar tendinopathy: inflammatory mechanism, painful collagen breakage, mechanical impingement of the fat pad and activation of the nociceptors of the patellar fat pad by the locally released biochemical irritants. ${ }^{(2)}$ Chronic patellar tendinopathy is a clinical syndrome that is characterized by pain and tenderness at the inferior patellar pole and/or the proximal part of the patellar tendon and by tendon abnormalities on imaging (MRI, ultrasound). ${ }^{(3)}$ Professional athletes with a high training frequency in high impact sporting activities are especially affected by jumper's knee, which can result in persistent pain and functional limitation. This can influence training, competition, and performance levels and may lead to the end of a career for top athletes in some cases. ${ }^{(4)}$

Conservative treatment has been proposed as the first therapeutic approach by using rest, anti-inflammatory treatment, improving training techniques, athletic shoes, and physiotherapy with stretching and eccentric strengthening exercises. ${ }^{(5)}$

Unfortunately a large proportion of elite athletes do not respond to conservative treatment and after a prolonged period of persisting symptoms surgical treatment is indicated. ${ }^{(6)}$ While traditional open treatment has been applied consistently, arthroscopic procedures have been introduced promising better results than open methods, suggesting that they can better manage and more accurately target the painful areas of the proximal- posterior tendon fibres and of the infrapatellar fat pad. ${ }^{(7)}$

The aim of the study was to evaluate the results of arthroscopic management of cases with chronic refractory patellar tendinopathy (jumper's knee).

\section{Patients}

In this prospective study 28 sportive complaining of patellar tendinosis resistant to at least 3 months of conservative treatment, were managed by arthroscopic treatment in the period from May 2o18 to June 2o19. The patients mean age 23.25 range 18 -3o years. All patients were followed up for a period of mean 10.5 months range $9-12$ months. This 
study was done at Kafr Elsheikh and Benha university hospitals. Preoperative consent was taken from all patients. The study was approved by ethical committee at Kafr Elsheikh University and Benha University.

The age of patients included in this study were ranging from 18 to 30 years with the mean age of $23.25 \pm 3.36$ years. All patients had active sport participation. All patients subjected to conservative treatment for at least 3 months (physiotherapy, non-steroidal and steroid anti-inflammatory drugs). Patients with concomitant intra or extraarticular knee joint pathologies (i.e. patellofemoral malalignment/maltracking, chondral lesions, meniscal tears and ligamentous injuries) were excluded from the study.

All patients in the study were males. Right knee was affected in 17 cases and left in 11 cases. All patients were unilaterally affected. Most of the patients were students, two patients were working as engineers, one as a waiter and other as a worker. All patients involved in sports activities football 9 (32.1\%), basketball 9 (32.1\%), volleyball 6 $(21.4 \%)$ and handball $4(14.3 \%)$.

\section{Methods}

This study was done in the period between May 2o18 and June 2o19 where all patients included reported that they were subjected to a period of at least 3 months of conservative treatment in the form of relative rest, functional rehabilitation consists of analgesic and anti-inflammatory measures in association with mechanical physiotherapy consisting of eccentric strengthening and specific stretching exercises, injection of corticosteroids or platelet-rich plasma. But, without relief of symptoms and were managed arthroscopically.

All patients were admitted in the ordinary ward and history was taken to assure functional activity. Full workup including clinical examination, radiological evaluation as well as laboratory investigations, was done for every patient. Radiological investigations include anteroposterior (AP) view, lateral view and skyline views were taken to ensure normal patellofemoral joint geometry of the knee joint for each patient.

MRI for assessment of the following criteria: $\mathrm{BME}$ of the inferior patellar pole, infrapatellar (IFP) oedema, IFP bursitis and thickening of the proximal patellar tendon. US and coloured duplex were done to evaluate fibre arrangement and to look for hypoechoic areas indicating pathology. CD-US is performed to assess presence of neovascularization.

All the patients were evaluated by the same examiner. The VAS, Lysholm knee scale and VISA-P scores were used to evaluate preoperative score. 
All patients complained for anterior knee pain located in the most proximal part of the patellar tendon, which presented severe local tenderness. Pain was present during whole practice and symptoms prevented all patients from participating in their usual highdemanding sports activities.

The mean duration of the symptoms before the patients undergone surgical treatment was $13 \pm 3.8$ months on average. The diagnosis of patellar tendinopathy was basically clinical. The main clinical finding was pain on palpation of the lower pole of the patella. Another finding from the physical examination was quadriceps muscle wasting.

All patients underwent US, coloured duplex and MRI to aid diagnosis and surgical planning. Criteria used for diagnosis were assessment of the following criteria: BME of the inferior patellar pole, IFP oedema, infrapatellar bursitis and thickening of the proximal patellar tendon at MRI, and hypoechoic regions corresponding to tendon tenderness at US. Also coloured Doppler was used to assess intratendinous neovascularization.

\section{Surgical technique:}

All patients were operated upon in supine position ordinary table with a thigh holder. High thigh pneumatic tourniquet was applied to the affected limb for all patients then preparation and draping of the affected limb was applied. In the procedure, a $4.0 \mathrm{~mm}$ and $30^{\circ}$ high definition arthroscope, a $4.5 \mathrm{~mm}$ straight shaver blade, a $4.0 \mathrm{~mm}$ straight acromianizer type burr, and a radiofrequency probe, were used.

All patients were operated on by the same surgeon using the same surgical technique. The area of maximal tenderness at the patellar tendon was marked preoperatively and in all patients matched the abnormalities seen on MRI and US. The surgical approach began with routine arthroscopic examination of the knee to exclude coexistent pathologic lesions using an anterolateral portal for the arthroscope, the anteromedial portal for instruments

After visualization of the entire joint with the $30^{\circ}$ arthroscope, an accessory SL portal is created. In typical fashion, an outside-toinside needle technique is used under direct arthroscopic inspection to obtain optimal portal placing. The knee was kept extended with the arthroscope in one of the anterior (AM or AL) portals and the desired instrumentation was introduced through the other corresponding portal.

Focal synovectomy was performed using a shaver and an electrocautery device around the inferior patellar pole and the proximal and posterior tendon portion. Once the extraarticular part of the inferior patellar pole that 
was covered by the patellar tendon enthesis (bone-tendon interface) was clearly visualized, a needle was inserted to coronally extend the lesion estimated using preoperative MRI.We also used a needle to aid location of the apex of the patella. Demonstrating the view obtained with a $30^{\circ}$ arthroscope from the SL portal, the proximal part of the patellar tendon is identified and visualized throughout its entire width.

This view allows for clear identification of the inflamed, pathologic tissue and facilitates its removal up to healthy tendon margins. Furthermore, by palpating the patellar tendon from the outside and inserting a needle at its lateral and medial borders, it is easier to demarcate and define the exact width of the tendon, which can sometimes be obscured by the inflamed synovial tissue and the fat pad.

Arthroscopic debridement of the adipose tissue of the Hoffa's body posterior to the dorsal surface of the patellar tendon, debridement of the abnormal portion of the deep surface of the proximal patellar tendon, and arthroscopic excision of the lower pole of the patella if needed (presence of a prominent bony spur in preoperative radiology). (Figs $1,2)$

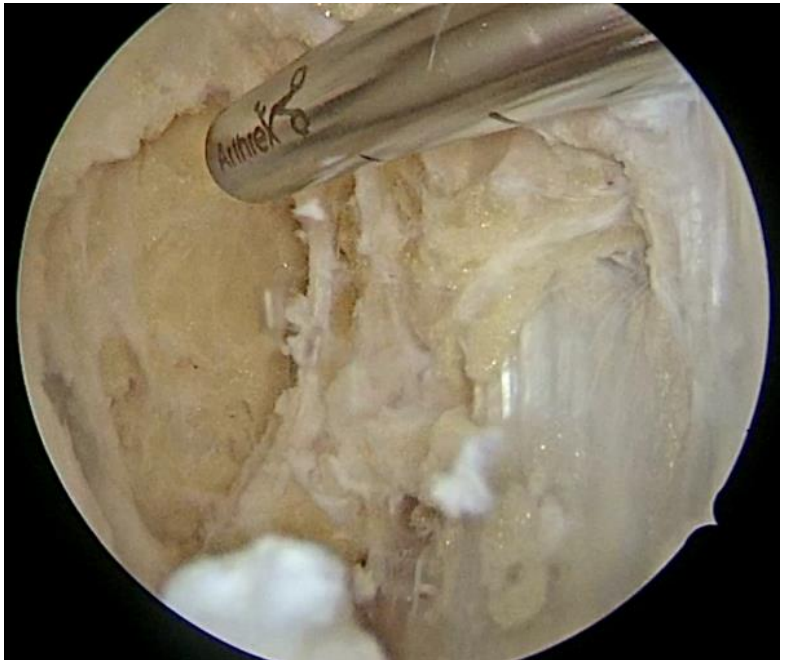

Fig 1: Debridement the most proximal Hoffa fat pad.

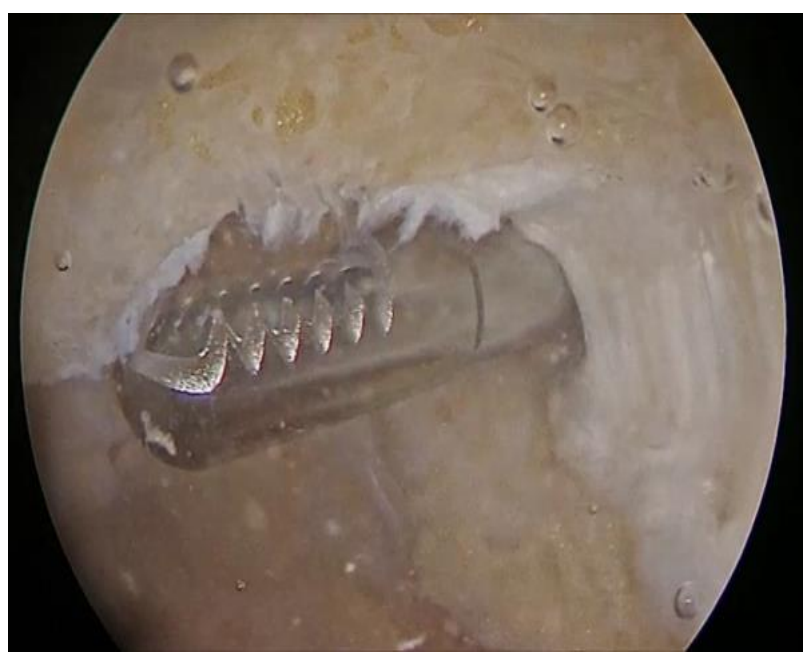

Fig 2: Arthroscopic appearance of the inferior patellar pole after abrasion

All patients were allowed for partial weight bearing with crutches from day one. The patients were instructed to start full nonweight bearing range of motion exercises. After one week walking and light bicycling activity were initiated. Light concentric and eccentric strength training for the quadriceps muscles was instituted. 
During the second week after treatment, the patients were told to gradually increase their tendon loading activity with more sport specific training. Running typically was allowed after six weeks. Patients were scheduled to be evaluated postoperatively for a period of mean 10.5 months range 9-12 months.

No patients were lost at follow-up. The Lysholm knee scoring system, the Victorian Institute of Sport Assessment VISA-P score and VAS scale were used for the assessment of the pre-operative condition and the postoperative outcome on the given time intervals.

\section{Results}

This work involved the study of a prospective case series of 28 patients and follow up for a period of mean 10.5 months range 9-12 months to assess the effect of arthroscopic management of cases with chronic refractory patellar tendinopathy (jumper's knee).

The patients included in the study were admitted to Kafr Elsheikh and Benha University Hospitals with chronic refractory patellar tendinopathy after at least 3 months of conservative treatment, they were treated with arthroscopic patellar tendon debridement and shaving.
The mean age was $23.25 \pm 3.36$ (range 18 -3o years). They were all (28) males. Patients' demographics are illustrated in table 1.

Table 1: patients' demographics

\begin{tabular}{lll}
\hline Sport activity type & $\mathbf{N = 2 8}$ & Percentage \\
\hline Football & 9 & 32.1 \\
Basketball & 9 & 32.1 \\
Volleyball & 6 & 21.4 \\
Handball & 4 & 14.3 \\
Gender & & \\
Male & 28 & 100 \\
Female & 0 & 0 \\
Side & & \\
Right & 17 & 61 \\
Left & 11 & 39 \\
Age (years) & & \\
Min-Max & $18-30$ & \\
Mean \pm SD & $23.26 \pm 3.36$ \\
\hline
\end{tabular}

The mean preoperative VISA-P score was $38.4 \pm 4.1$ points, range $30-47$ points. The mean preoperative VAS score was $38.4 \pm 4$.1points, range 6-9 points. The mean preoperative LYSHOLM score was $52.4 \pm 6$ points, range $42-62$ points. The mean duration of pain was $13 \pm 3.8$ months, range $8-18$ months. All patients were operated upon after a period of conservative treatment of mean $6.5 \pm 1$ months, range 5-8 months.

At last follow-up, all outcome measures showed significant improvements compared to their preoperative values. There was highly significant improvement of VAS score from (mean $7.39 \pm 1.06$ points, range: $6-9$ points) preoperatively to (mean $0.39 \pm 0.99$ points, 
range: o-5 points) at last follow up. This difference was statistically highly significant (Z: $-4.67, \mathrm{p}<0.00)$.

There was highly significant improvement of VISA score from (mean $38.36 \pm 4$.1points, range: $30-47$ points) preoperatively to (mean $93.43 \pm 7$ o points, range: $5-1$ oo points) at last follow up. This difference was statistically highly significant (Z: -4.63, p <o.oo). (Fig 3)

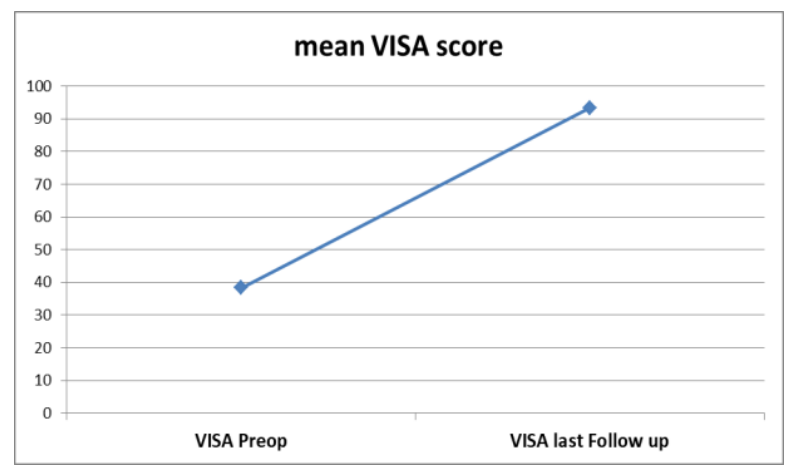

Fig 3: The difference between preoperative and last follow up VISA score

There was highly significant improvement of Lysholm score from (mean $52.42 \pm 5.9$ points, range: $42-62$ points) preoperatively to (mean, 94.6 \pm 6.6 , range: $75-100$ points) at last follow up. This difference was statistically highly significant (Z: 4.51, $\mathrm{p}<0.00)$.

The mean time period required for return to preinjury sports activity was $3.9 \pm 1.07$ months (range, 2.5- 7 months). At follow-up, 27 patients $(96.4 \%)$ were able to perform at their preinjury sports activities. Patients were classified into 2 groups according to the period to return to sports. Group A $(n=22$, $78.6 \%$ ) returned to sport at 5 months or less.
They did not experience any symptoms during or after sports activity. Group $B(n=5$, $17.9 \%$ ) needed more than 5 months to return to sports. They felt minor to moderate pain after intense sports performance. One case was not able to return to preinjury sports activity. He complained from notable pain at the onset sports activity. He was a male, 27 years old. He works at restaurant and he usually had to ascend and descend stairs in his job, also poor compliance to the rehabilitation program could be the cause.

\section{Discussion}

Chronic patellar tendinopathy is a relative common source of pain in sports involved populations. The cause is presumably stress overload, particularly in sports with high incidence of jumps that result in proximal patellar tendon micro tears that affect the tendon-bone junction causing chronic tendinopathy. ${ }^{(8)}$

Patellar tendinopathy frequently affects sports practice, even forcing some athletes to end their careers. Different training methods and an adequate physical exercise program often improve athletes' performance, although symptoms generally take at least 3 months to improve. $^{(9)}$

Conservative treatment is usually the initial therapeutic approach to chronic patellar tendinopathy. ${ }^{(10)}$ 
Although chronic patellar tendinopathy commonly resolves with non-operative treatment and changes in training methods, in some patients, symptoms persist, thus raising the possibility of surgery. The surgery can be approached via open and arthroscopic approaches. The results from this study suggested that arthroscopic debridement of the adipose tissue of the Hoffa's body posterior to the patellar tendon and debridement of abnormal patellar tendon, with shaving the insertion of the tendon to the patellar apex, was effective in improving pain, subjective daily function, and sports activities with minimal risk of postoperative complications.

Compared to open approach, the arthroscopic procedures can provide faster recovery and rehabilitation, high rates of return to full sports activities, and excellent clinical results in terms of pain reduction and function in athletes whose conditions are refractory to conservative treatment. The arthroscopic procedures also approach chronic patellar tendinopathy via soft tissue alone or combined with a bony procedure. ${ }^{(11)}$

Open procedures are designed to debride the bone and tendon involved in the pathology. Excision of the inferior extra articular patellar pole and reattachment of the patellar tendon were reported. Ferreti et al. (2oo2) reported relief of pain with bony resection and central patellar tendon excision in 33 patients with 8year mean follow up with 7o\% "excellent" and $15 \%$ "good results. ${ }^{(12)}$

Early rehabilitation and return to sports is a possible advantage of the arthroscopic technique, making it particularly attractive for professional athletes However, in a study comparing open versus arthroscopic techniques by 2 different surgeons, the arthroscopically treated patients returned to sports at 6 months compared with 1o months for the open technique. ${ }^{(13)}$

It was suggested that all of the patients treated by open method returned to their former activities, but the authors presented eight knees in seven patients and they reported $86 \%$ " excellent" and 14\% "fair results. (14)

There are different techniques described for arthroscopic treatment of chronic patellar tendinosis. The technique in this study consists of the debridement of soft tissue of the proximal patellar tendon and the inferior patellar pole region, partial resection of the Hoffa fat pad, and cauterization of local neovascularization.Also additional bony resection of the inferior patellar pole if needed.

A prospective study including 15 athletes was presented in 2006 where an arthroscopic technique for treatment of chronic patellar tendinopathy, was used. Hypertrophic 
synovitis around the inferior patellar pole was removed, and a release of the paratenon and a bone denervation at the inferior patellar pole were done without removal of any tendon or bone material. ${ }^{(15)}$

Willberg \& colleagues (2007) treated patients with an arthroscopic shaving of the dorsal side of the proximal patellar tendon. ${ }^{(16)}$

Coleman and other co-workers described shaving of the affected tissue along with excision of part of the infrapatellar fat pad. ${ }^{(17)}$

Alaseirlis and colleagues (2o12) performed an osteoplasty of the distal patellar pole, debridement of the Hoffa fat pad and of degenerated tissue of the proximal posterior patellar tendon, and cauterization of the visible neovessels. ${ }^{(18)}$

In this study, all patients were athletes with refractory chronic patellar tendinopathy that was unsuccessfully treated conservatively for a minimum of 3 months. The results of the present study was that arthroscopic patellar tendon debridement and decortication of the inferior pole of the patella showed good to excellent symptomatic improvement in the professional athletes with anterior knee pain due to refractory chronic patellar tendinopathy. The VISA-P scores improved from $38.36 \pm 4.1$ to $93.34 \pm 7.28$, the lysholm scores improved from $52.42 \pm 5.9$ to $94.6 \pm 6.6$,
Lorbach and others (20o8) performed arthroscopic resection of the lower patellar pole of $2 \mathrm{o}$ patients with chronic patellar tendinosis with unsuccessful conservative treatment. They found excellent clinical results with fast rehabilitation and with a significant improvement in the Lysholm score, Kujala score, visual analog scale for pain, function, and satisfaction 6 weeks postoperatively.They reported no postoperative complications after arthroscopic debridement in 20 patients. ${ }^{(19)}$

Pascarella and collegues (2o11) showed that the VISA-P score was significantly improved (from 35.3 to 69.8 at the one-year stage and to 70.7 at the three-year follow-up). Their surgical indication was persistent patellar tendinopathy despite a minimum of three months of nonoperative treatment, and $42 \%$ were professional athletes. ${ }^{(7)}$

Alaseirlis and others (2o12) found that the patients showed a major improvement in the mean Lysholm score as well as in the mean Victorian Institute of Sports AssessmentPatellar (VISA-P) score at the 1oth postoperative week. ${ }^{(15)}$

In this study, $96.5 \%$ of the athletes were able to return to full sports activities after 4 months. only one case was not able to return to preinjury sports activity, he complained from notable pain at the onset sports activity. We suggested that his work at café and he 
usually had to ascend and descend stairs in his job could be the cause of not return to his previous level of performance activity also poor compliance to the rehabilitation program.

Romeo and Larson reported $100 \%$ returned to their previous physical activity level, but this study only reported two patients, one with a follow up of 2 months and the other 2 years. They return within 8 weeks after surgery. ${ }^{(20)}$

Coleman and others pointed out symptomatic benefits in $96 \%$ of arthroscopic tenotomy patients, four months faster return to preinjury level compared with the open treatment group. ${ }^{(17)}$

A prospective study by ogon et al. (20o6), found that within 3 months after surgery, 13 of 15 patients were free of symptoms; 14 of 15 athletes reached their preinjury level of sporting activities. ${ }^{(15)}$

Willberg and others (2007) found that all patients returned to sports within 3 months postoperatively. They concluded that arthroscopic resection of the lower patellar pole provides a fast return to sporting activities in patients with chronic patellar tendinopathy. ${ }^{(16)}$

Lorbach and other researchers (20o8) found that $75 \%$ of the patients reached their preinjury level of activity after 6 months. At 2-year follow-up, the results showed an improvement of the Tegner activity level, with 19 of 20 patients reaching their former level of activity and 18 of 20 patients with good to excellent results in the modified classification for jumper's knee of Blazina. (19)

Pascarella and co-workers (2o11) found that $70.3 \%$ of the athletes were able to return to their sport at the same level by three months postoperative. ${ }^{(7)}$

Maier and collaegues (2o13) reported that, after arthroscopic patellar tendon release within the symptomatic zone in 35 competitive athletes, $76.7 \%$ were able to return to sports at previous levels by 4.4 months. ${ }^{(21)}$

This study had some limitations. First, it was a case series, lacked a control group, and did not compare the results with other techniques. Second, the number of patients was relatively small. Third, follow up period was short. A more extended study with control group, large number of patients and long term follow up are recommended.

\section{Conclusion}

Arthroscopic patellar debridement is a minimally invasive operative technique for the treatment of chronic patellar tendinopathy (jumper's knee) resistant to conservative 
treatment. It appears to provide significant improvements in symptoms and function. The results of this study suggest that patients are able to achieve their previous sporting level. The low surgical impact to the patellar tendon allows an early and functional rehabilitation. Compared with previous surgical techniques, this technique is effective and safe.

\section{References}

1. Blazina ME, Kerlan RK, Jobe FW, Carter VS, Carlson GJ. Jumper's knee. Orthop Clin North Am. 1973;4:665-678.

2. Fredberg U, Stengaard-Pedersen K. Chronic tendinopathy tissue pathology, pain mechanisms, and etiology with a special focus on inflammation, Scand J Med Sci Sports 2oo8; 18: 3-15.

3. Roels J, Martens M, Mulier JC, Burssens A. Patellar tendinitis (jumper's knee). Am J Sports Med. 1978; 6:362-368.

4. Lian OB, Engebretsen L, Bahr R. Prevalence of jumper's knee among elite athletes from different sports: a crosssectional study. Am J Sports Med 20o5;33:561-567.

5. Visnes H, Bahr R. The evolution of eccentric training as treatment for patellar tendinopathy (jumper's knee): a critical review of exercise programs. Br J Sports Med 2oo7; 41:217-223.

6. Kaeding CC, Pedroza AD, Powers BC. Surgical treatment of chronic patellar tendinosis: a systematic review. Clin Orthop Relat Res 2007; 455:1o2-106.

7. Pascarella A, Alam M, Pascarella F, Latte C, Di Salvatore MG, Maffulli N. Arthroscopic management of chronic patellar tendinopathy. Am J Sports Med 2o11;39(9):1975-1983.
8. Sharma P, Maffulli N. Tendon injury and tendinopathy: healing and repair. J Bone Joint Surg Am. 2oo5;87:187-2o2.

9. Bahr R, Fossan B, Løken S, Engebretsen L. Surgical treatment compared with eccentric training for patellar tendinopathy (jumper's knee) a randomized, controlled trial. J Bone Joint Surg Am. 2oo6; 88:1689-1698.

10. Nanos KN, Malanga GA. Treatment of patellar tendinopathy refractory to surgicalmanagement using percutaneous ultrasonic tenotomy and platelet-rich plasma injection: a case presentation. PM R 2o15;7(12):130o-13o5.

11. Santander J, Zarba E, Iraporda H, Puleo S. Can arthroscopically assisted treatment of chronic patellar tendinopathy reduce pain and restore function? Clin Orthop Relat Res 2o12;47o(4):993997.

12. Ferretti A, Conteduca F, Camerucci E, Morelli F. Patellar tendinosis: a follow-up study of surgical treatment. J Bone Joint Surg Am. 2oo2;84:2179_ 2185.

13. Coleman B, Khan K, Kiss Z, Bartlett J, Young D, Wark J. Open and arthroscopic patellar tenotomy for chronic patellar tendinopathy: a retrospective outcome study. Am J Sports Med 2ooo; 28(2): 183-19o.

14. Griffiths G, Selesnick H. Operative treatment and arthroscopic findings in chronic patellar tendinitis. Arthroscopy. 1998;14: 836-839.

15. Ogon P, Maier D, Jaeger A, Suedkamp NP. Arthroscopic patellar release for the treatment of chronic patellar tendinopathy. Arthroscopy 20o6;22:462.e1-462.e5.

16. Willberg L, Sunding K, Ohberg L, Forssblad M, Alfredson H. Treatment of Jumper's knee: promising short term results in a pilot study using a new arthroscopic approach based on imaging findings. Knee Surg Sports Traumatol Arthrosc 20o7; 15(5): 676-681. 
17. Coleman BD, Khan KM, Maffulli N, Cook JL, Wark JD. Studies of surgical outcome after patellar tendinopathy: clinical significance of methodological deficiencies and guidelines for future studies. Victorian Institute of Sport Tendon Study Group. Scand J Med Sci Sports 2000; $10(1): 2-11$.

18. Alaseirlis DA, Konstantinidis GA, Malliaropoulos N, Nakou LS, Korompilias A, Maffulli N. Arthroscopic treatment of chronic patellar tendinopathy in high-level athletes. Muscles Ligaments Tendons J 2o12;2(4):267-272.

19. Lorbach O, Diamantopoulos A, Paessler $\mathrm{H}$. Arthroscopic resection of the lower patellar pole in patients with chronic patellar tendinosis. Arthroscopy 2oo8;24 (2):167-173.

20. Romeo AA, Larson RV. Arthroscopic treatment of Infrapatellar tendonitis. Arthroscopy 1999;15:341345.

21. Maier D, Bornebusch L, Salzmann GM, Sudkamp NP, Ogon P. Mid- and long-term efficacy of the arthroscopic patellar release for treatment of patellar tendinopathy unresponsive to nonoperative management. Arthroscopy 2o13;29(8):1338-1345.

To cite this article: Ahmed R. Elzeiny, Adel H. Adawy, Hatem E. Elgohary, Saad A. Shoulah. Arthroscopic Treatment of Chronic Patellar Tendinopathy "Jumper's Knee". BMFJ 2020; 37(3):500-511, DOI: 10.21608/bmfj.2020.15570.1031. 